\title{
Hydrodynamic Analog for Electromagnetic Structures of Sprites
}

\author{
K. H. W. Chu \\ Department of Physics, Xinjiang University, Wulumuqi 830046, China \\ Email: chukh04@xju.edu.cn
}

\begin{abstract}
Since the discovery by Franz [1], sprites have now been observed over thunderstorms all over the world. The first columniform sprites (c-sprites) reported were vertical columns of light which may extend about from 76 to $87 \mathrm{~km}$ and probably less than $1 \mathrm{~km}$ in diameter [2]. Upon using the hydrodynamic analog we can derive some families of stationary Beltrami field-like solutions from the free Maxwell equations in vacuum.

The Beltrami condition [3], a representation of the alignment of a vorticity with its flow, describes the simplest and perhaps the most fundamental equilibrium state in the system of vortex dynamics. These stationary electromagnetic fields are helical and/or column-like once they are represented in a suitable frame of reference. Possible dendritic and jelly-fish-like patterns of sprites are demonstrated.
\end{abstract}

\section{Acknowledgements}

The author is partially supported by the Starting Funds for 2005-XJU Scientific Research Scholars.

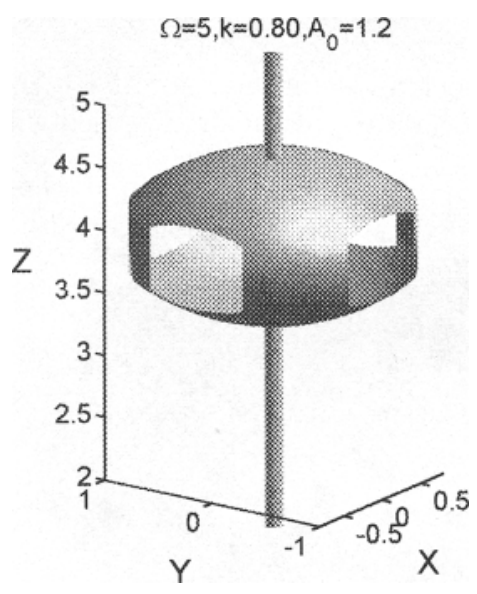

Figure : Possible sprite- and column-like electromagnetic field.

\section{REFERENCES}

1. Franz R C, Nemzek R J, Winckler J R. Television image of a large upward discharge above a thunderstorm system. Science, 1990; 249: 48-51

2. Sentman D D, Wescott E M, Osborne D L, et al. Preliminary results from the Sprites94 campaign: Red Sprites. Geophys Res Lett, 1995; 22: 1205-1208

3. Chu R K H. Possible ball-like formations of the Beltrami flow field. Meccanica, 2004; 39: 181-186 